\title{
A Study of Bending Mode Algorithm of Adaptive Front-Lighting System Based on Driver Preview Behavior
}

\author{
Zhenhai Gao ${ }^{1,2}$ and Yang $\mathrm{Li}^{1}$ \\ ${ }^{1}$ State Key Laboratory of Automobile Simulation and Control, Jilin University, Changchun 130022, China \\ ${ }^{2}$ State Key Laboratory of Vehicle NVH and Safety Technology, Changan Automobile Holding, Chongqing 401120, China \\ Correspondence should be addressed to Yang Li; 89271667@qq.com
}

Received 25 February 2014; Accepted 4 April 2014; Published 4 May 2014

Academic Editor: Hamid Reza Karimi

Copyright (c) 2014 Z. Gao and Y. Li. This is an open access article distributed under the Creative Commons Attribution License, which permits unrestricted use, distribution, and reproduction in any medium, provided the original work is properly cited.

\begin{abstract}
The function of adaptive front-lighting system is to improve the lighting condition of the road ahead and driving safety at night. The current system seldom considers characteristics of the driver's preview behavior and eye movement. To solve this problem, an AFS algorithm modeling a driver's preview behavior was proposed. According to the vehicle's state, the driver's manipulating input, and the vehicle's future state change which resulted from the driver's input, a dynamic predictive algorithm of the vehicle's future track was established based on an optimal preview acceleration model. Then, an experiment on the change rule of the driver's preview distance with different speeds and different road curvatures was implemented with the eye tracker and the calibration method of the driver's preview time was established. On the basis of these above theories and experiments, the preview time was introduced to help predict the vehicle's future track and an AFS algorithm modeling the driver's preview behavior was built. Finally, a simulation analysis of the AFS algorithm was carried out. By analyzing the change process of the headlamp's lighting region while bend turning which was controlled by the algorithm, its control effect was verified to be precise.
\end{abstract}

\section{Introduction}

AFS (adaptive front-lighting system) is a front-lighting system that can change the light pattern and illumination area according to the vehicle's state such as the velocity, the steering wheel angle, and road environment to light the road ahead effectively so as to reduce accidents at night. According to AFS productions of international companies and research institutes' research findings and directions currently, the classic algorithm of AFS can be divided into the following four categories.

(1) The AFS Algorithm Based on the ECER123 Regulation [1]. The basic demand of this algorithm was to control the deflection angle of the headlamp at an allowed range of the regulation. Strambersky et al. (European Lighting Technology Centre of Visteon Company) used Ackerman steering geometry principle to calculate the headlamp's deflection angle in the regulation's allowed range [2]. The algorithm based on the regulation needed to be improved in accuracy and trajectory prediction.

(2) The AFS Algorithm Based on Brake Safety. The algorithm used the stopping sight distance to calculate arc length and the stopping sight distance was larger than brake distance to make sure that the driver would observe the obstacle in front. Huang, Lifu and Qian, Yu, Deng, Mclaughlin et al., and Rong et al. had used similar methods in their studies [3-8]. The design based on brake safety could ensure the safety, but the headlamp's lighting location had a lag relative to the vehicle's location.

(3) The AFS Algorithm Based on Early Lighting. When a car was driven on the curve, the headlamp should be lighted on the location where the car would arrive at after a while. This kind of research solved the problem to some extent that the headlamp illumination direction had a lag compared to the driving direction $[9,10]$. This kind of AFS can be divided into nonpredictive AFS and predictive AFS. 
(a) Nonpredictive AFS. Koji Ishiguro and Yuji Yamada who were in Denso and Toyota in Japan studied the relationship between vehicles' speed and drivers' fixation distance during bend driving in the day time. They concluded that the fixation distance got larger while the speed increased. By studying drivers' reaction time in dangerous circumstances such as collision, they regarded $t=3 \mathrm{~s}$ as an appropriate time which they used in their AFS algorithm [11]. Nonpredictive AFS could improve the lighting condition of the road ahead to some extent, but it could only use the current steering wheel angle and velocity to estimate the vehicle's transient current trajectory and then calculate the headlamp's deflection angle. When the road's curvature changed a lot, the beam might deflect too slow in the corner and even deflected to a wrong direction at an S-curve.

(b) Predictive AFS. By GPS, electronic map, CCD which perceived the traffic environment information including vehicle locations, road types, lane numbers and road curvatures, and vehicles' state information which was used to predict cars' future track, the P-AFS controlled the headlamp in advance. Ibrahim of Visteon applied for the patent which was called "Predictive adaptive front lighting algorithm for branching road geometry," Patent number US 7558822 B2 [10]. They matched the GPS information with the database of electronic map to confirm the vehicle's current location and predict the road's information such as road type, lane numbers, and curvature and road branches and finally calculated the headlamp deflection angle by P-AFS algorithm. Kim et al. in Korea (2010) proposed a new method of PAFS which was called curvature estimated swivel in their paper [12]. By using the existing devices such as LDWs to replace the GPS to predict road curvature, the headlamp's deflection angle was calculated. Predictive AFS solved the nonpredictive AFS's error problem to some extent when the road's curvature changed a lot, but it did not take the driver's visual characteristics into consideration.

(4) The AFS Algorithm Based on the Driver's Vision in the Corner. Many researchers have studied the driver's sight movement in a bend or crossing [13-15]. Although these researches aimed to study the driver's fixation behavior while not providing information for the development or design of AFS, they had some guiding significances for the design of AFS.

On the basis of these experiments, Young et al. used the regular pattern of heads' turning angle at daytime and night to guide the design of AFS [16], but the paper did not mention the specific algorithm of AFS and the precision could not be ensured because of the limit of measuring devices.

Considering the regulation and safety, the algorithm of AFS currently estimated the vehicles' future driving track according to vehicles' moving states, traffic environment, and drivers' wheel input. Then the AFS algorithm controlled the headlamp's deflection angle to illuminate the estimated driving track in advance. But most of these algorithms assumed that the speed and the wheel angle would not change and assumed the driving track as a circle. According to Zhang et al.s study on vehicles' lateral moving characteristics [17-19], the possible variation of vehicles' longitude and lateral speed was not considered which would also result in the change of driving track. In some circumstances such as in a curve entry, in a curve exit in an irregular road, or the driver's acceleration or deceleration to pass a varying curve, the algorithm could not predict the future driving track well and the effect of AFS was not very well.

Meanwhile, as the most important active safety device, the headlamp's main effect is to provide illumination for the road ahead. Hence, the AFS should satisfy the safety demand, ensure that the driver's concerned region is fully illuminated, and must not influence the driver's fixation behavior. Its illumination effect also has a direct relationship with the driver's eye comfort. Compared to a good environmental illumination condition such as in the daytime, it should not additionally increase the driver's visual fatigue. In this aspect, although some researches have used the statistic rule of drivers' visual field to control the headlamp's deflection, in the practical application, this method which was totally based on the statistic rule cannot ensure the safety.

Based on the considerations above, an AFS algorithm considering the driver's preview behavior was proposed. The vehicle's kinematics and dynamics characteristics were used comprehensively to calculate the vehicle's future track which was more reliable than the method purely based on the vehicle's state information the headlamp's deflection lag and direction error was avoided. Under the premise of ensuring safety, parameters of the driver's fixation behaviors were introduced to increase the measuring precision of the driver's visual statistical rule by using the eye tracker and the headlamp's illumination is more in compliance with the driver's fixation behavior. This paper proposes an original technology route; the work was first carried out in early 2010, and it has obtained national invention patents [20].

\section{AFS Algorithm Considering the Driver's Preview Behavior}

2.1. AFS Algorithm. By modeling the driver's preview behavior at a bend, the vehicle's future track in a period of time was predicted according to the vehicle's current state and the vehicle's steady state response characteristics to the driver's wheel, gas pedal, or brake pedal input. Simultaneously, the driver's fixation location on the future track was determined according to drivers' preview behavior rule (the rule of fixation location or preview spot) at real bends; then the headlamp's deflection angle was controlled and the location was illuminated effectively. The technical route of the algorithm was shown in Figure 1.

The algorithm simulated the driver's preview behavior at a large curvature bend and an integral algorithm to predict the vehicle's track was proposed. The algorithm was based on the hypothesis of steady preview and dynamic correction [21]. According to the vehicle's current state and its possible change which resulted from the driver's inputs, the vehicle's future track was predicted by the algorithm [22]. As Figure 2 showed, the vehicle's coordinate system at current time $t$ was the reference coordinate system. The preview time $t_{p}$ was 


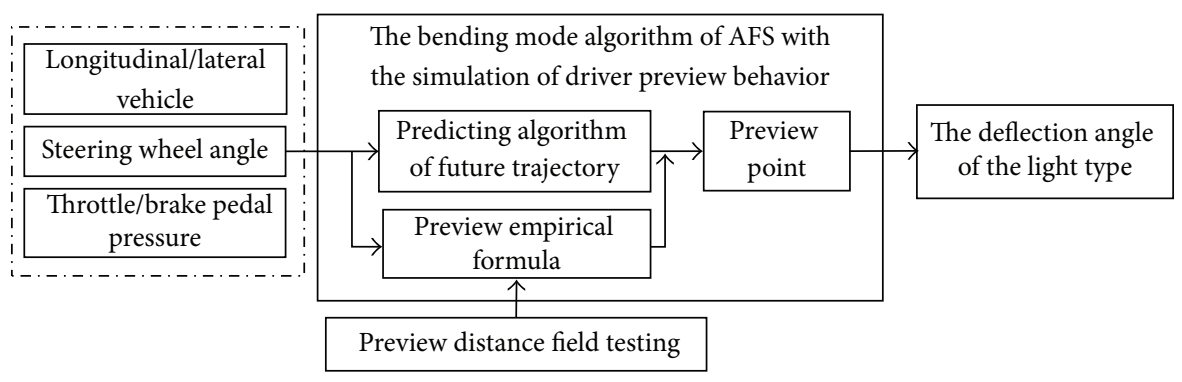

FIgURE 1: The specific technical route.

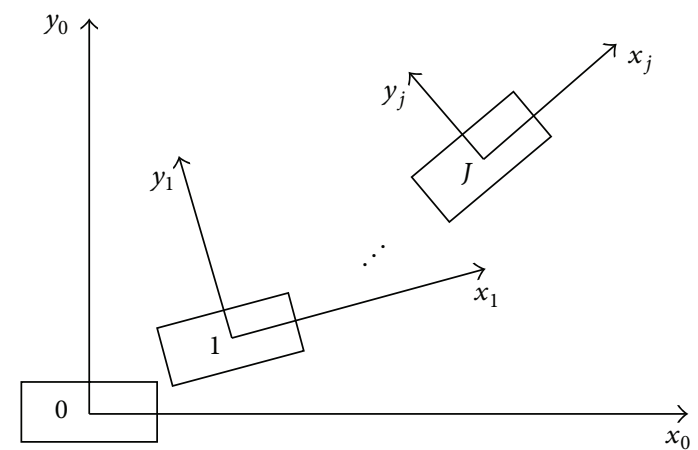

FIGURE 2: The coordinate system.

evenly divided into $J$ pieces $\left(\Delta t_{p}=t_{p} / J\right)$. During the preview time, the longitudinal and lateral acceleration which resulted from the driver's wheel or gas pedal input were the same.

Because every time piece is quite short, the mutual effect of the vehicle's longitudinal and lateral movement can be neglected. On the basis of the vehicle's state (location, velocity, and acceleration) at the initial moment of the time piece, the vehicle's state at the end of the time piece was calculated using the rigid body kinematics principle [23]. Then from the moment $t$, every time piece's vehicle state could be calculated. As (1) showed (at the time piece $j$, the initial moment is $t_{j}-1$ and the end moment is $t_{j}$ ), the combination of the location at every time formed the vehicle's future track in $t_{p}$ time:

$$
\begin{gathered}
{\left[\begin{array}{c}
\dot{x}_{j} \\
\dot{y}_{j}
\end{array}\right]=\left[\begin{array}{c}
\dot{x}_{j-1} \\
\dot{y}_{j-1}
\end{array}\right]+\left[\begin{array}{c}
\ddot{x}_{j} \\
\ddot{y}_{j}
\end{array}\right] \times \Delta t_{p},} \\
{\left[\begin{array}{c}
x_{j} \\
y_{j}
\end{array}\right]=\left[\begin{array}{c}
x_{j-1} \\
y_{j-1}
\end{array}\right]+A_{j-1,0}\left(\left[\begin{array}{c}
\dot{x}_{j-1} \\
\dot{y}_{j-1}
\end{array}\right] \times \Delta t_{p}+\frac{1}{2}\left[\begin{array}{c}
\ddot{x}_{j} \\
\ddot{y}_{j}
\end{array}\right] \times \Delta t_{p}^{2}\right) .}
\end{gathered}
$$

The coordinate transformation matrix is

$$
A_{j 0}=\left(\begin{array}{cc}
\cos \varphi_{j} & -\sin \varphi_{j} \\
\sin \varphi_{j} & \cos \varphi_{j}
\end{array}\right) .
$$

$\varphi$ is path angle which is calculated by

$$
\varphi_{j}=\varphi_{j-1}+\frac{\ddot{y}_{j}}{\dot{x}_{j}} \cdot \Delta t_{p} .
$$

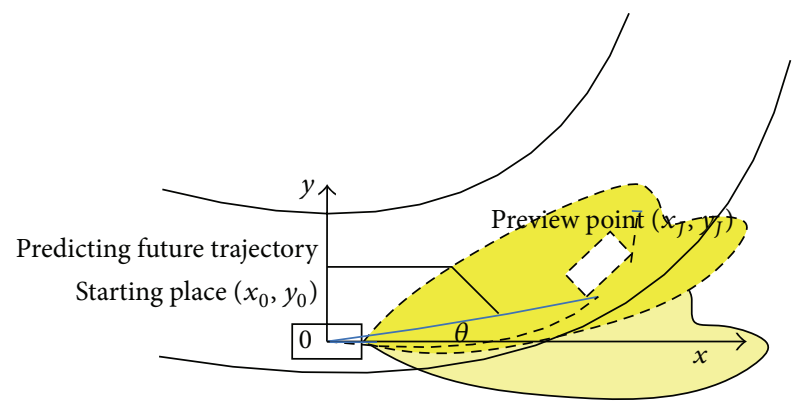

Figure 3: Adaptive front-lighting system deflection angle calculation mathematical model.

$j=1,2, \ldots, J ;\left(x_{j}, y_{j}\right),\left(\dot{x}_{j}, \dot{y}_{j}\right)$, and $\left(\ddot{x}_{j}, \ddot{y}_{j}\right)$ were the vehicle's location, the vehicle's velocity, and the vehicle's steady acceleration at time $t_{j} ; \varphi_{j}$ was the vehicle's course angle at time $t_{j}$. The vehicle's coordinate system at current time $t$ $\left(t_{0}\right)$ was the reference coordinate system. During the preview time, the longitudinal and lateral acceleration which resulted from the driver's wheel or gas pedal input were the same.

Then the vehicle's mass centre coordinate $\left(x_{j}, y_{j}\right)$ after the preview time $t_{p}$ can be calculated at the coordinate system of current time $t$. As Figure 3 showed, the point was the driver's preview location at current time $t$ and the headlamp's deflection angle $\theta$ which was the angle between the vehicle's predicted location and the vehicle's longitudinal axis was calculated by

$$
\theta=\arctan \frac{y_{J}-y_{0}}{x_{J}-x_{0}} .
$$

Based on the above theory, the measurement of the preview distance for real drivers was conducted. By the advanced eye tracker, the driver's fixation behavior was recorded when the driver drove through the bend. The driver's gazing direction was analyzed and the average value of the preview distance was calculated. Then an empirical equation of the relationship between preview distance, preview time and velocity, and road curvature was proposed and the preview time for the AFS algorithm was modified. 
TABLE 1: The experiment velocity and the curvature radius.

\begin{tabular}{lc}
\hline Radius of curvature $(\mathrm{m})$ & Velocity $(\mathrm{km} / \mathrm{h})$ \\
\hline 20 & $10,15,20,25,30$ \\
30 & $20,30,40$ \\
40 & $20,30,40$ \\
\hline
\end{tabular}

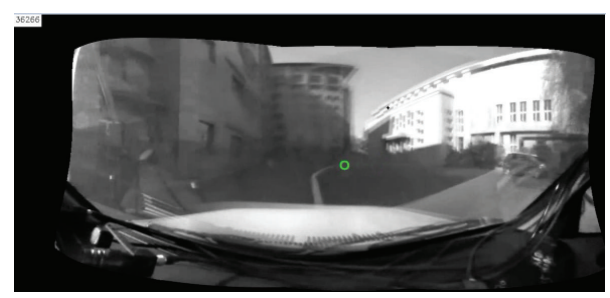

Figure 4: Driver's gaze point position comparison chart when "being close to the bend."

\section{The Experiment Measurement and Change Rule Study on the Driver's Preview Distance}

\subsection{Experiment Measurement}

3.1.1. Experiment Equipment. SmartEye Pro eye tracker (sample frequency: $60 \mathrm{~Hz}$ ), A JETTA car made by FAWVolkswagon, a $12 \mathrm{~V}$ spare battery, meter ruler, the adhesive tape to make the lane, and others.

3.1.2. Experiment Conditions. Eight drivers with normal vision. Three quarter-circle lanes with curvature radius of $20 \mathrm{~m}, 30 \mathrm{~m}$, and $40 \mathrm{~m}$ were used as the experiment road. With every lane, the experiment was done for three times repeatedly. Before the formal experiment, every driver did 10minute exercise. The experiment velocity and the curvature radius were shown in Table 1.

3.1.3. The Definition of Fixation Location. The SmartEye Pro eye tracker recorded the driver's raw eye movement data. According to the thresholds of eye movement parameters set up by the users, the fixation and saccade behaviors were confirmed. Meanwhile, by the corresponding software, the video of the driving scene was replayed and the fixation location (the green circle in Figure 4) was spotted on the driving scene.

The threshold settings of eye movement were as follows:

(1) the threshold of visual angle deviation: 2 deg.;

(2) the eyeball movement velocity threshold during fixation: $15^{\circ} / \mathrm{s}$, which was the highest velocity of eyeball movement during a fixation;

(3) the eyeball movement velocity threshold during saccade: $35^{\circ} / \mathrm{s}$, which was the lowest velocity of eyeball movement during a saccade;

(4) the fixation duration threshold: $200 \mathrm{~ms}$.

According to the road environment, the state change of the vehicle, the fixation location (visual angle), and its change

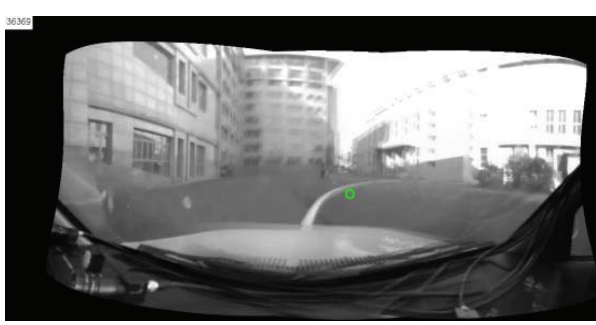

(a)

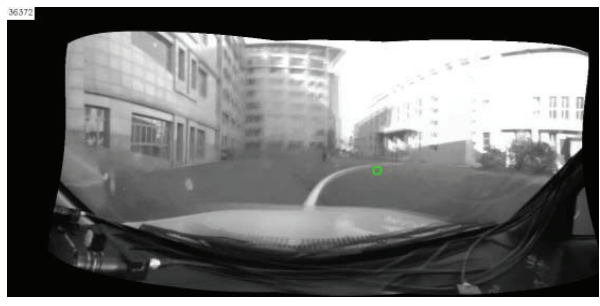

(b)

FIGURE 5: Driver's gaze point position comparison chart before and after into the corner: (a) is before into the corner, and (b) is after.

in the driving scene video, the entire driving process was divided into four sections: "straight lane," "being close to the bend," "entering the bend," and "being out of the bend."

"Straight lane": in this section, the entire bend was shown in the driver's visual field. Repeated experiments showed that the driver would sweep the bend and form a rough impression of the available track; then the fixation location would be a few meters away from the vehicle as shown in Figure 4.

In the section of "being close to the bend," because the vehicle's movement state had not changed, the nonpredictive AFS algorithm could not predict the bend ahead and activate the AFS bend lighting function.

"Entering the bend": as shown in Figure 5 (there were three frames intervals between two images which meant the time interval was $0.05 \mathrm{~s}$ ). There was a saccade in almost all experiments when the driver just drove from the "being close to the bend" section to the "entering the bend" section. It was also shown in the driver's eye movement data that there was a saccade in the moment. This saccade behavior was defined as the boundary between the "being close to the bend" section and the "entering the bend" section. After this saccade, the lane area was defined as the "entering the bend" section.

In the "entering the bend" section, the driver needed to steer and the AFS needed to activate bend lighting function and control the headlamp's deflection according to some rules. So according to the purpose of this experiment and our algorithm's parameters' need, this section was the emphasis of our study.

"Being out of the bend": the driver would brake when he were going to be out of the bend. This section would not be analyzed in detail.

3.1.4. The Preview Distance Calculation and the Analysis of Its Changing Rule. The preview distance was calculated in the 


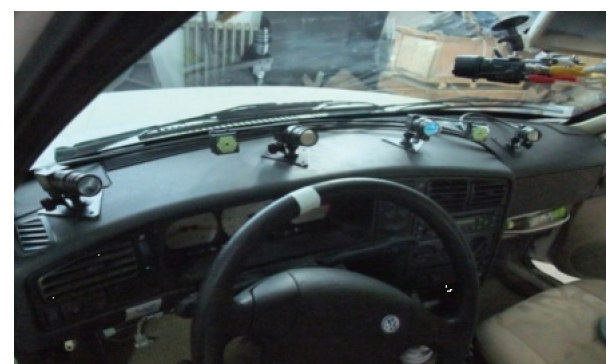

FIGURE 6: The eye tracking system's installation location.

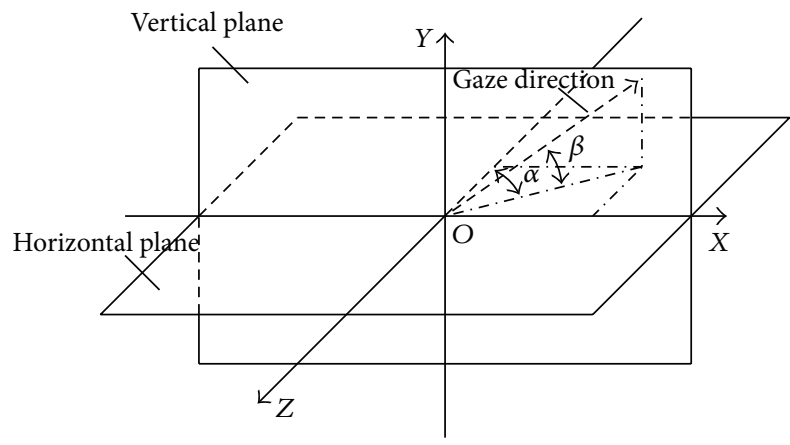

FIGURE 7: The world coordinate system and gaze direction angle.

"entering the entrance" section during the driving process. The fixation point in the front near the centre line of the road was defined as the preview point. The SmartEye Pro system was installed as shown in Figure 6. The definition of the SmartEye Pro world coordinate system was as follows: the connection line between number 2 camera and number 3 camera in SmartEye Pro system was the $X$-axis of the world coordinate, the midpoint of the connection line was the coordinate origin, and the $Y$-axis was in the vertical plane. The coordinate system was shown in Figure 7.

As shown above, the angle $\alpha$ between the gaze direction's projection in XOZ plane and the negative direction of the world coordinate's $Z$-axis (the positive direction of the vehicle coordinate's $X$ direction) was the gaze direction's horizontal direction angle. The angle $\beta$ between the gaze direction and the horizontal plane was the gaze direction's vertical angle.

The gaze direction's horizontal angle is

$$
\alpha=\arctan \frac{\text { GazeDirection. } x}{- \text { GazeDirection. } z} .
$$

The gaze direction's vertical angle is

$$
\beta=\arcsin \text { GazeDirection. } z \text {. }
$$

GazeDirection. $x(y, z)$ was the projection of the unit vector along the gaze direction on the $X(Y, Z)$ axis of the world coordinate system.

According to the gaze direction and the gaze origin in the world coordinate system, the preview point's coordinate could be calculated in the world coordinate system as shown in Figure 8. The distance away from the driver which was the preview distance could be calculated by (7) (the vehicle's roll

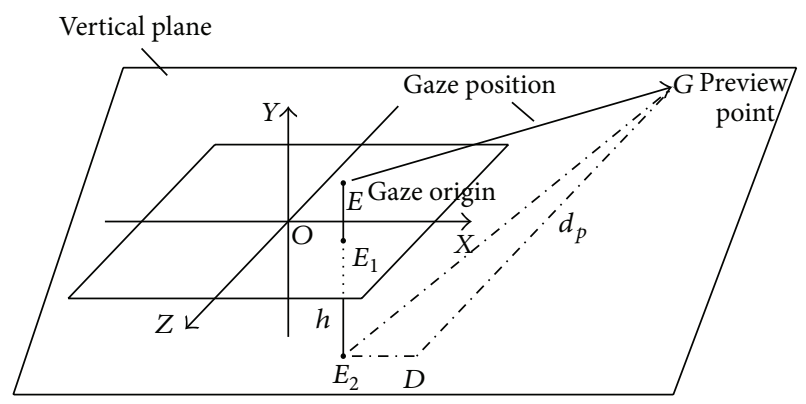

Figure 8: Preview distance calculation schematic.

TABle 2: Preview distance data under $40 \mathrm{~m}$ bend radius.

\begin{tabular}{lccccc}
\hline \multirow{2}{*}{ Data operation } & \multicolumn{5}{c}{ Bend radius $(20 \mathrm{~m})$} \\
& $10 \mathrm{~km} / \mathrm{h}$ & $15 \mathrm{~km} / \mathrm{h}$ & $20 \mathrm{~km} / \mathrm{h}$ & $25 \mathrm{~km} / \mathrm{h}$ & $30 \mathrm{~km} / \mathrm{h}$ \\
\hline Mean & 9.6253 & 9.7248 & 9.8515 & 10.0431 & 10.1059 \\
Variance & 1.0292 & 0.9282 & 0.8827 & 0.8319 & 1.2074 \\
Maximum & 7.5492 & 7.7634 & 8.0599 & 8.0124 & 8.2775 \\
Minimum & 12.3558 & 12.0545 & 12.1912 & 12.4505 & 12.1334 \\
\hline
\end{tabular}

TABle 3: Preview distance data under $20 \mathrm{~km} / \mathrm{h}$ velocity.

\begin{tabular}{lcc}
\hline Data operation & \multicolumn{2}{c}{ Velocity $(20 \mathrm{~km} / \mathrm{h})$} \\
\hline Mean & $30 \mathrm{~m}$ & $40 \mathrm{~m}$ \\
Variance & 9.4637 & 11.3121 \\
Maximum & 1.8896 & 1.4886 \\
Minimum & 6.6242 & 7.6275 \\
\hline
\end{tabular}

and pitch movement were neglected). The detailed data of the preview distance was shown in Tables 2 and 3 :

$$
d_{p}=(\text { GazeOriginy }+h) \frac{\text { GazeDirection. } z}{\text { GazeDirection. } y} .
$$

$h$ is the vertical height of the world coordinate system's origin away from the ground; GazeOrigin. $y$ is the $y$ coordinate of the gaze origin; GazeDirection. $z$ is the $z$ coordinate of the unit vector along the gaze direction; GazeDirection. $y$ is the $y$ coordinate of the unit vector along the gaze direction.

When the road curvature radius was $20 \mathrm{~m}$, the preview distance increased slowly from $9,6253 \mathrm{~m}$ to $10.1059 \mathrm{~m}$ with the adding of the velocity. When the velocity was constantly $20 \mathrm{~km} / \mathrm{h}$, the driver's preview distance increased too while the road curvature radius increased from $30 \mathrm{~m}$ to $40 \mathrm{~m}$. Noh et al. had used the preview time and the response of neuromuscular system as main human factors to study the preview distance at different velocities and different road curvatures whose conclusion was similar to ours [24].

The experiment's result was consistent with the previous test's conclusion. With the consideration of the characteristics of the preview distance's distribution and change rule, the preview distance-velocity regression analysis was done to get the preview distance-velocity empirical equation (9). At the speed of $20 \mathrm{~km} / \mathrm{h}$, according to the preview distance data with 
the curvature radius of $30 \mathrm{~m}$ and $40 \mathrm{~m}$, the preview distancecurvature radius empirical equation (10) was gotten by liner fitting. The fitting line is

$$
y=0.025 x+9.358
$$

$y$ is the preview distance; $x$ is velocity; $R$ is the correlation coefficient, $R 2=0.98082$, and the observed value $r=$ 0.99036. $n=5$; with the significance level $a=0.05, r=$ $0.99036>r 0.05(n-2)=0.878$; the regression result was obvious and the hypothesis "the velocity change will affect the driver's preview distance" was confirmed. To be consistent with previous described parameters, the empirical equation $(r=20 \mathrm{~m})$ was rewritten as follows:

$$
d_{p}=0.025 v+9.358, \quad r=20 \mathrm{~m},
$$

where $v$ is velocity, $\mathrm{km} / \mathrm{h}, r$ is curvature radius, and $d_{p}$ is the preview distance.

At the speed of $20 \mathrm{Km} / \mathrm{h}$, the empirical equation of the preview distance-curvature radius was as follows:

$$
d_{p}=0.185 r+1.529, \quad v=20 \mathrm{~km} / \mathrm{h} .
$$

According to the relationship between the preview time and the preview distance, $d_{p}=v t_{p}$, the empirical equation of the preview time was as follows:

$$
\begin{array}{cl}
t_{p}=0.09+\frac{33.689}{v}, & r=20 \mathrm{~m}, \\
t_{p}=0.0333 r+0.2752, & v=20 \mathrm{~km} / \mathrm{h} .
\end{array}
$$

According to (11), the preview time calculated under the speed of $10 \mathrm{~km} / \mathrm{h}, 15 \mathrm{~km} / \mathrm{h}, 20 \mathrm{~km} / \mathrm{h}, 25 \mathrm{~km} / \mathrm{h}$, and $30 \mathrm{~km} / \mathrm{h}$ was $3.489 \mathrm{~s}, 2.336 \mathrm{~s}, 1.774 \mathrm{~s}, 1.438 \mathrm{~s}$, and $1.213 \mathrm{~s}$, respectively; the average preview time is $1.70 \mathrm{~s}$. According to (12), the average preview time is $1.44 \mathrm{~s}$. Then (11) and (12) as empirical equations of the preview time simulating the driver's preview behavior were used to replace the fixed preview time tp in the AFS algorithm to confirm the location of the driver's preview point where the vehicle would arrive after the preview time. Then the headlamp's deflection angle could be calculated.

\section{The Simulation Analysis of the AFS Algorithm Simulating the Driver's Preview Behavior}

4.1. The Headlamp's Light Distribution. The optical analysis software LucidShape was used to simulate the headlamp's light distribution and acquire the headlamp's isolux curve on the ground. In the following simulation process, the envelope area of the isolux curve at a certain illumination was regarded as the headlamp's irradiation area. According to the relative relationship between the area and the road, the control effect of the algorithm was verified by the headlamp's irradiation effect. With the reference of ECE regulation, a dipped headlight in accordance with the ECE regulation was chosen in our simulation and its optical structure was not changed. The dipped headlight's location settings were as follows.

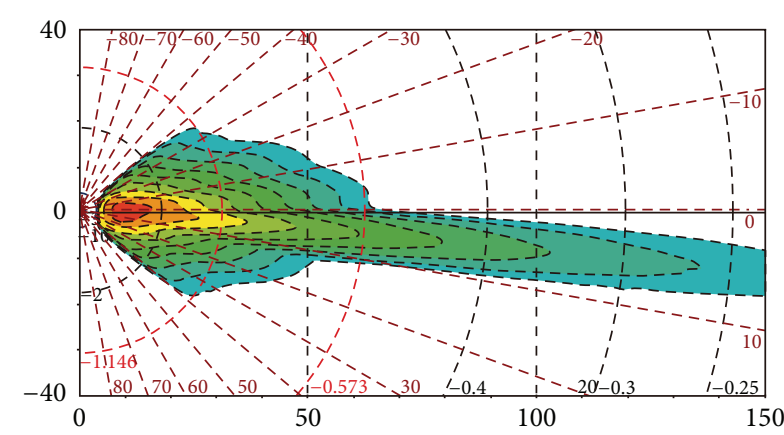

(m)

FIGURE 9: The simulation result of the headlamp's illumination on the road.

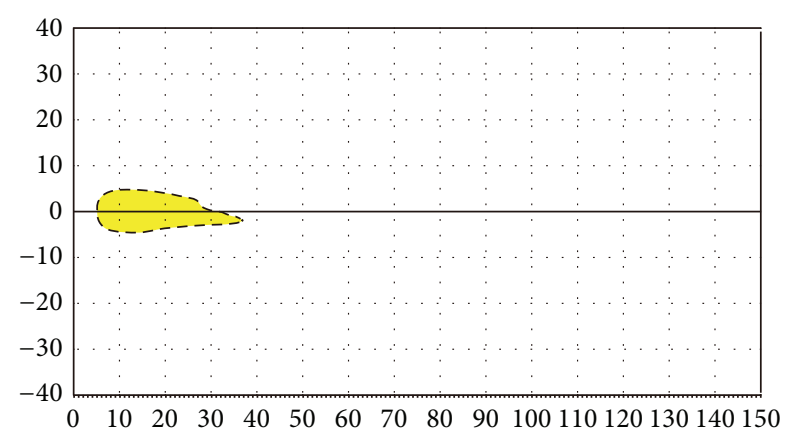

(m)

Figure 10: The envelope area of 321x isolux curve.

(1) The headlamps were installed in front of the vehicle and the headlamps were $1.2 \mathrm{~m}$ apart and were symmetrically on both sides of the vehicle's longitudinal axis.

(2) The installation height of headlamps was $0.65 \mathrm{~m}$.

The simulation result of the headlamp's illumination on the road was shown in Figure 9. The coordinate origin was the midpoint of the vehicle's forefront. The horizontal and vertical coordinates were horizontal and vertical locations relative to the vehicle (unit $\mathrm{m}$ ); different colors represented different illuminations.

Meanwhile, because the road's curvature radius was relatively small in our simulation, to clearly verify our algorithm's irradiation effect, the envelope area of 321x isolux curve was drawn in LabVIEW as the headlamp's irradiation area which was shown in Figure 10.

4.2. The Algorithm Verification. The vehicle model, driver model, and road model of CarSim were used in the simulation. The AFS algorithm and the real-time display of the headlamp's deflection angle, beam location, and irradiation effect were realized in LabVIEW. By the cosimulation of CarSim and LabVIEW, the bend driving simulating real environment was achieved. According to the calculated headlamp's deflection angle, irradiation area, and the vehicle's future track, the algorithm's control effect was verified. 


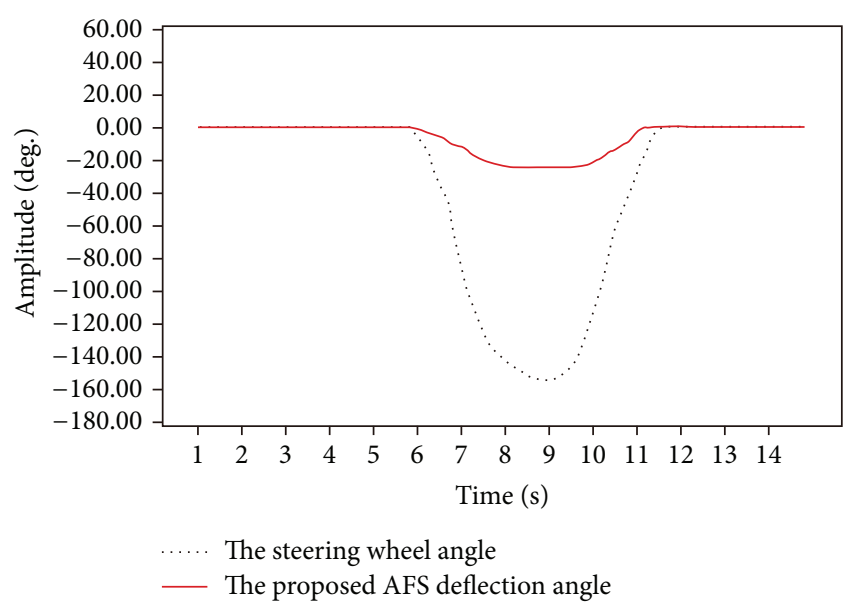

FIGURE 11: The headlamp's deflection angle and steering wheel angle.

4.2.1. Simulation Conditions and Parameters. The vehicle's speed was $30 \mathrm{~km} / \mathrm{h}$, the curvature radius of the bend was $20 \mathrm{~m}$, and the preview time $t_{p}(s)$ was as follows:

$$
t_{p}=0.09+\frac{33.689}{v}=1.213
$$

4.2.2. The Simulation Result Analysis. The headlamp's beam deflection angle of our algorithm was shown in Figure 11. The dotted line represented the wheel's steering angle and the solid line stood for the headlamp's deflection angle. It was shown that the deflection angle calculated by our algorithm could follow the wheel's steering angle very well. In other words, the algorithm could respond to the change of vehicle state quite well and controlled the headlamp's deflection angle. The control effect of the algorithm was shown in Figure 12. The dotted line represented the fixed headlamp beam.

It was shown in Figure 12 that the predicted track of our algorithm was close to the road's centre line which meant that the track prediction's accuracy was quite well. The vehicle's location on the predicted track after the preview time was used to guide the headlamp's deflection. The headlamp was controlled to deflect to the direction of the bend and the headlamp's irradiation area covered most areas of the front bend. When the vehicle was out of the bend, the algorithm could make the headlamp beam back on the nondeflection location to throw light on the front straight line.

\subsection{The Contrast Analysis of the Algorithm's Control Effect}

\subsubsection{The Simulation Conditions}

Condition 1. The initial speed was $20 \mathrm{~km} / \mathrm{h}$ and the driver accelerated to $30 \mathrm{~km} / \mathrm{h}$ in the time of $11 \mathrm{~s}-13 \mathrm{~s}$ and kept the velocity steady to pass through a half circle bend with the radius of $20 \mathrm{~m}$. The driving strategy was to make sure that the vehicle has no lateral offset relative to the lane's center line. The time parameter $t_{p}$ rose to $1.774 \mathrm{~s}$ from $1.213 \mathrm{~s}$ as follows:

$$
t_{p}=0.09+\frac{33.689}{v} .
$$

Condition 2. The driver drove through two continuous quarter circles (the radiuses were $30 \mathrm{~m}$ and $40 \mathrm{~m}$ ) at a constant speed of $20 \mathrm{~km} / \mathrm{h}$. The driver model used in the simulation was a model built-in CarSim. The time parameter $t_{p}$ rose to $1.607 \mathrm{~s}$ from $1.274 \mathrm{~s}$ as follows:

$$
t_{p}=0.0333 r+0.2752 .
$$

The contrast algorithm was a nonpredictive algorithm proposed by Ishiguro and Yamada which was relatively mature [11]. The main point of this algorithm is to control the headlamp's deflection to illuminate the location that the vehicle would arrive after $3 \mathrm{~s}$. The contrast simulation result was as follows.

\subsubsection{The Simulation Result Analysis}

Condition 1. The headlamp's deflection angle of the contrast algorithm and our algorithm was shown in Figure 15. The black dotted line was the wheel's steering angle curve, the solid line was the headlamp's deflection angle curve of our algorithm, and the dash dot line was the headlamp's deflection angle curve of the contrast algorithm.

It was shown in Figure 13 that both algorithms' headlamp deflection angle could follow the driver's steering angle. Relative to our algorithm, the contrast algorithm's headlamp deflection angle was about $20^{\circ}$ bigger at the moment $16 \mathrm{~s}$. The control effect of these two algorithms was shown in Figure 14 by the headlamps' irradiation area. The dotted line represented the fixed headlamp beam, the solid line represented the headlamp beam of our algorithm, and the long dashed line represented the headlamp beam of the contrast algorithm.

It was shown in Figure 14 that at the moment of $t=$ $10.19 \mathrm{~s}$, the vehicle just arrived at the bend; both the contrast algorithm and our algorithm could predict the vehicle's future track well and make the headlamp deflect to the direction of the bend. Their deflection angles were basically the same. Most areas of the front bend were covered by the central zone of the headlamp's irradiation region which meant that their illumination effects were basically the same.

At the moment of $t=12.27 \mathrm{~s}$, the vehicle was in the bend. The headlamp's deflection angle of the contrast algorithm was bigger than our algorithm which meant that its headlamp beam was more close to the inner side of the bend. Although the headlamp had illuminated more regions of the front bend, the control target of the contrast algorithm was to illuminate the location that the vehicle would arrive after $3 \mathrm{~s}$ while the driver's preview time was $1.213-1.774 \mathrm{~s}$ according to the empirical equation, so the region the driver cared for was a region which was close to the vehicle (the purple line in front of the vehicle) and it was not in the centre 

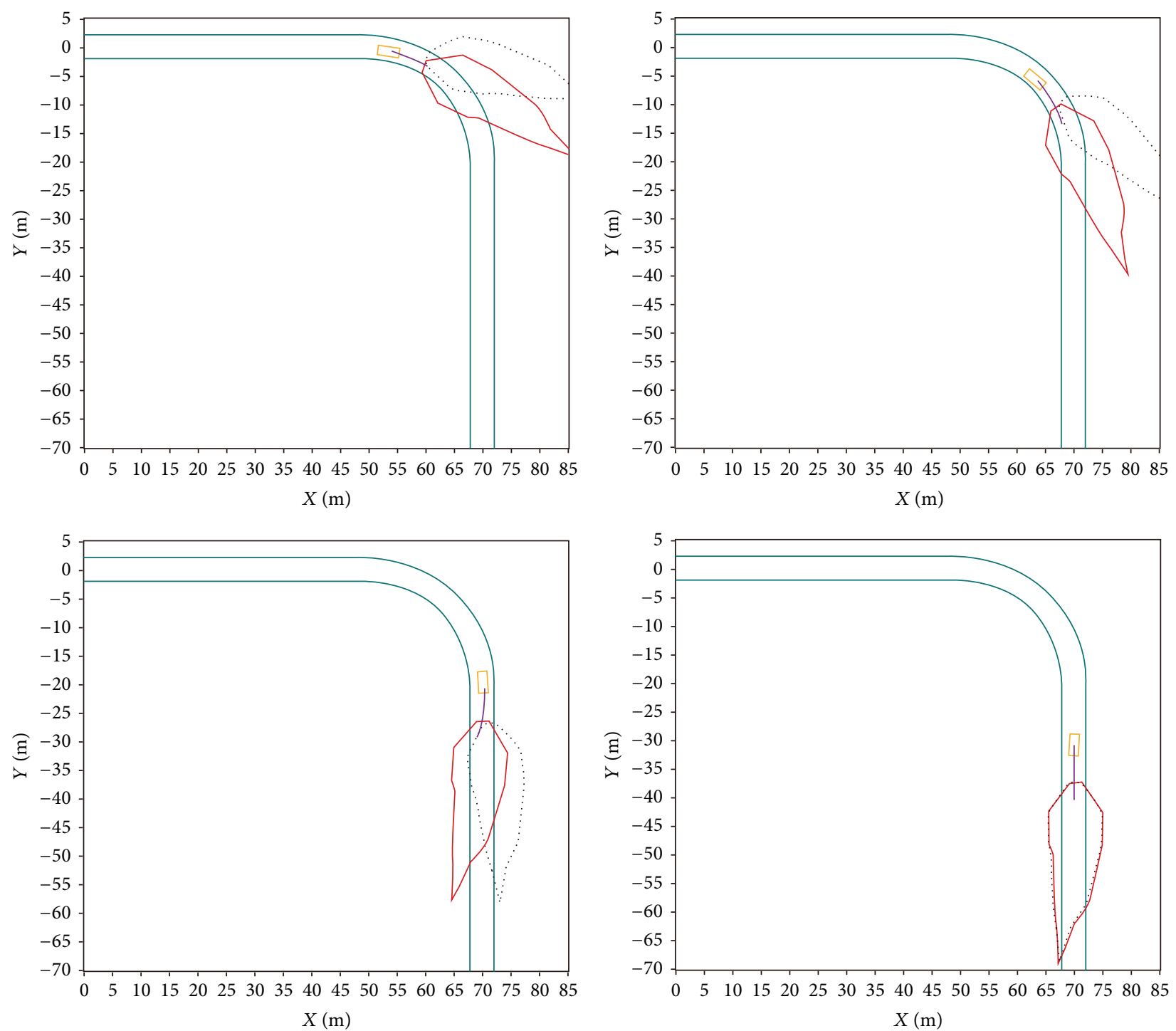

The fixed illumination shape

$\triangle$ This paper illumination shape

$\square$ The fixed illumination shape

$\triangle$ This paper illumination shape

FIGURE 12: The front-lighting illumination area simulation schematic.

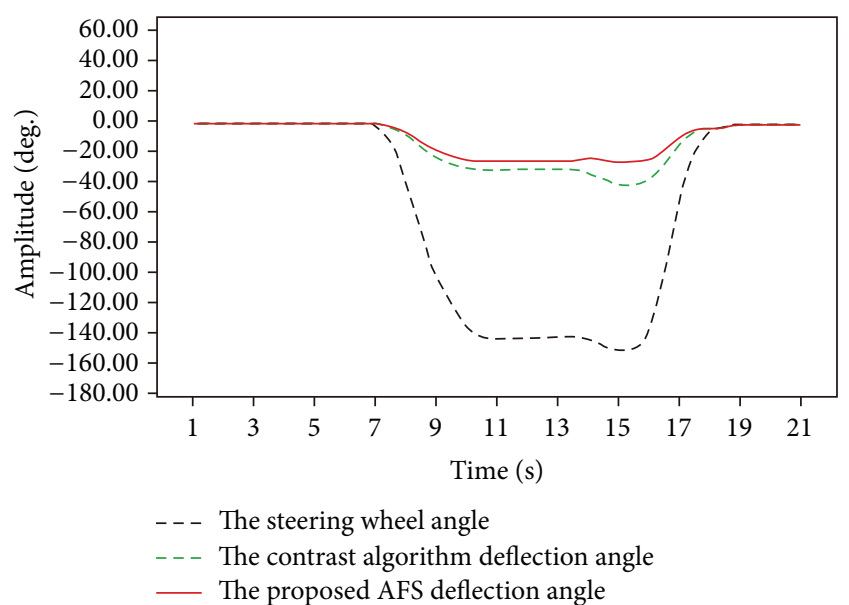

Figure 13: The headlamp's deflection angle and steering wheel angle (contrast algorithm and our algorithm diagram 1). of the contrast algorithm's illumination area which meant that its illumination effect was not well. The future track's prediction and irradiation region of our algorithm were more precise.

At the moment of $t=16.77 \mathrm{~s}$, the vehicle was going to be out of the bend. The headlamp's deflection angle of the contrast algorithm was bigger and its illumination effect was not ideal (relative to the fixed beam, its illumination effect could be worse). The future track's prediction of our algorithm was more precise and the headlamp's beam covered most of the front road which meant the illumination effect was relatively well.

At the moment of $t=18.86 \mathrm{~s}$, the vehicle was out of the bend. Both the contrast algorithm and our algorithm could quit from the bend illumination mode and control the headlamp back to the nondeflection location to ensure the straight 

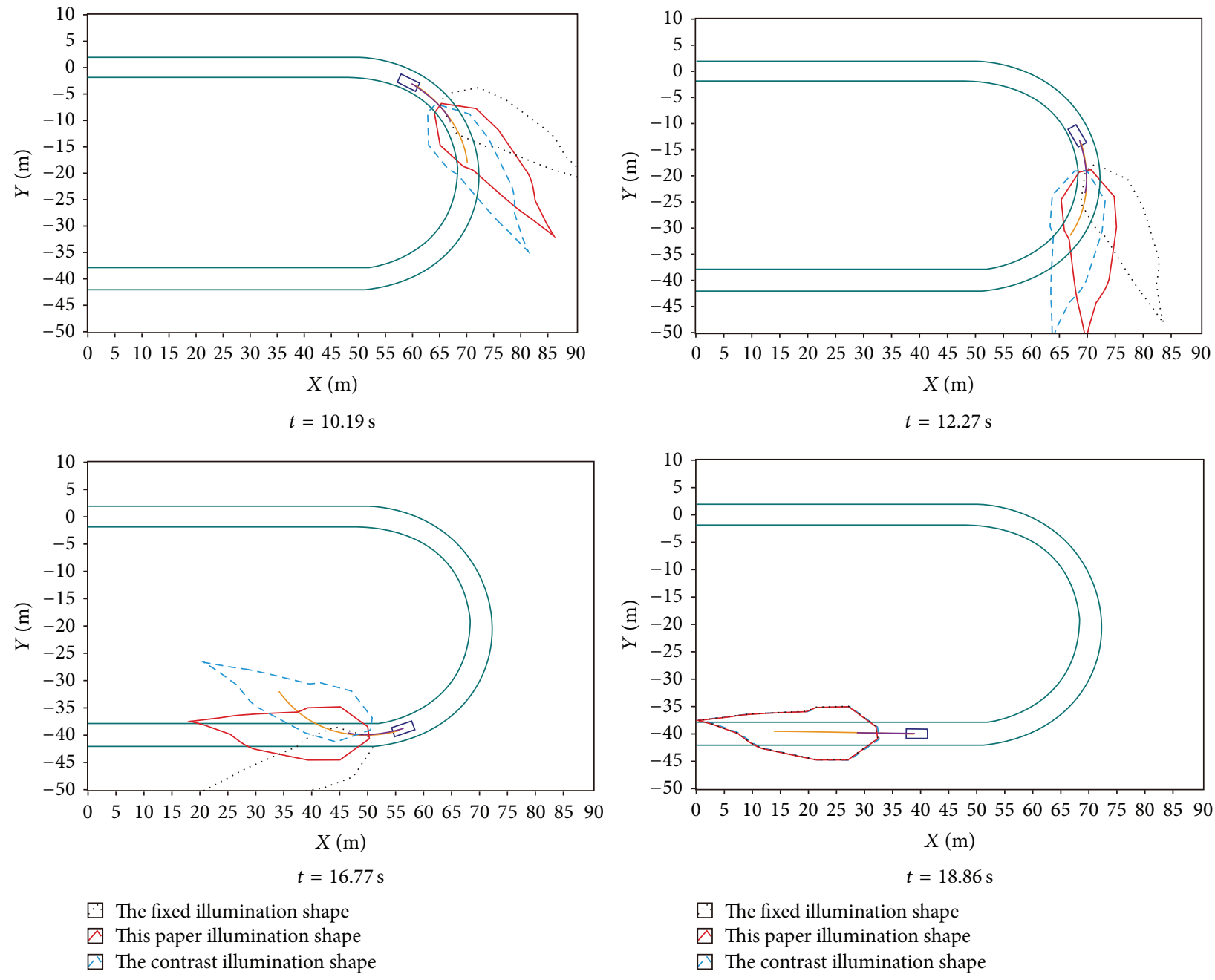

FIGURE 14: The front-lighting illumination area simulation schematic diagram 1.

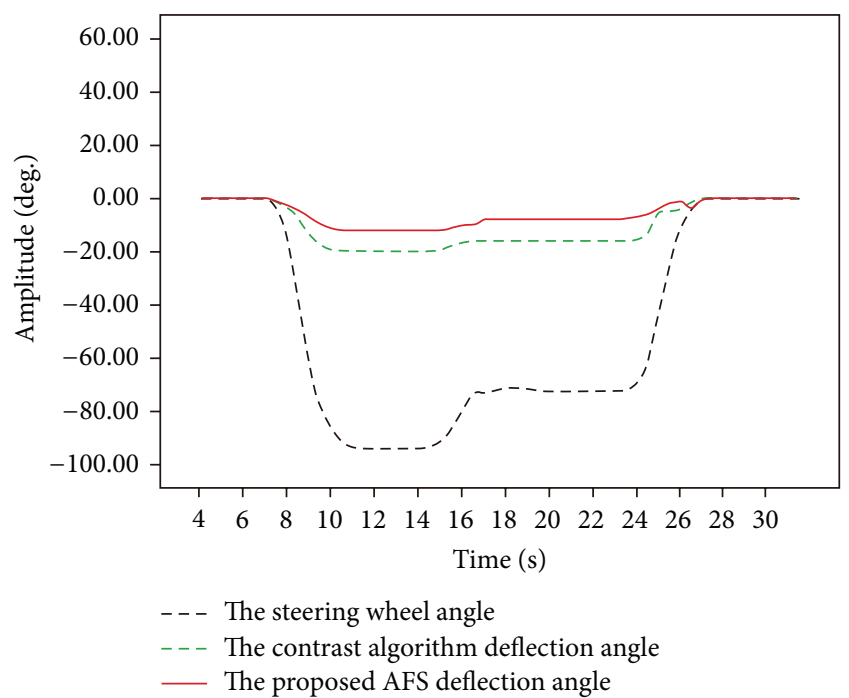

FIGURE 15: The headlamp's deflection angle and steering wheel angle (contrast algorithm and our algorithm diagram 2). line's illumination which meant that their illumination effects were the same.

Condition 2. The headlamp's deflection angle of the contrast algorithm and our algorithm was shown in Figure 15.

Both algorithms' deflection angle could follow the input of the driver's steering angle. Relative to our algorithm, the contrast algorithm's deflection angle was about $20^{\circ}$ bigger when the steering wheel began to deflect.

The specified control effect was shown in Figure 16 by the headlamp's illumination area. The explanation of the curves was similar to Figure 14 and the result was similar to Condition 1 which would not be analyzed again.

By the simulation result analysis of these two conditions, the conclusions were as follows.

(1) The future track's prediction of our algorithm was more precise (the purple line in the image during the entire simulation was more close to the lane's center line), so the algorithm would control the headlamp's 

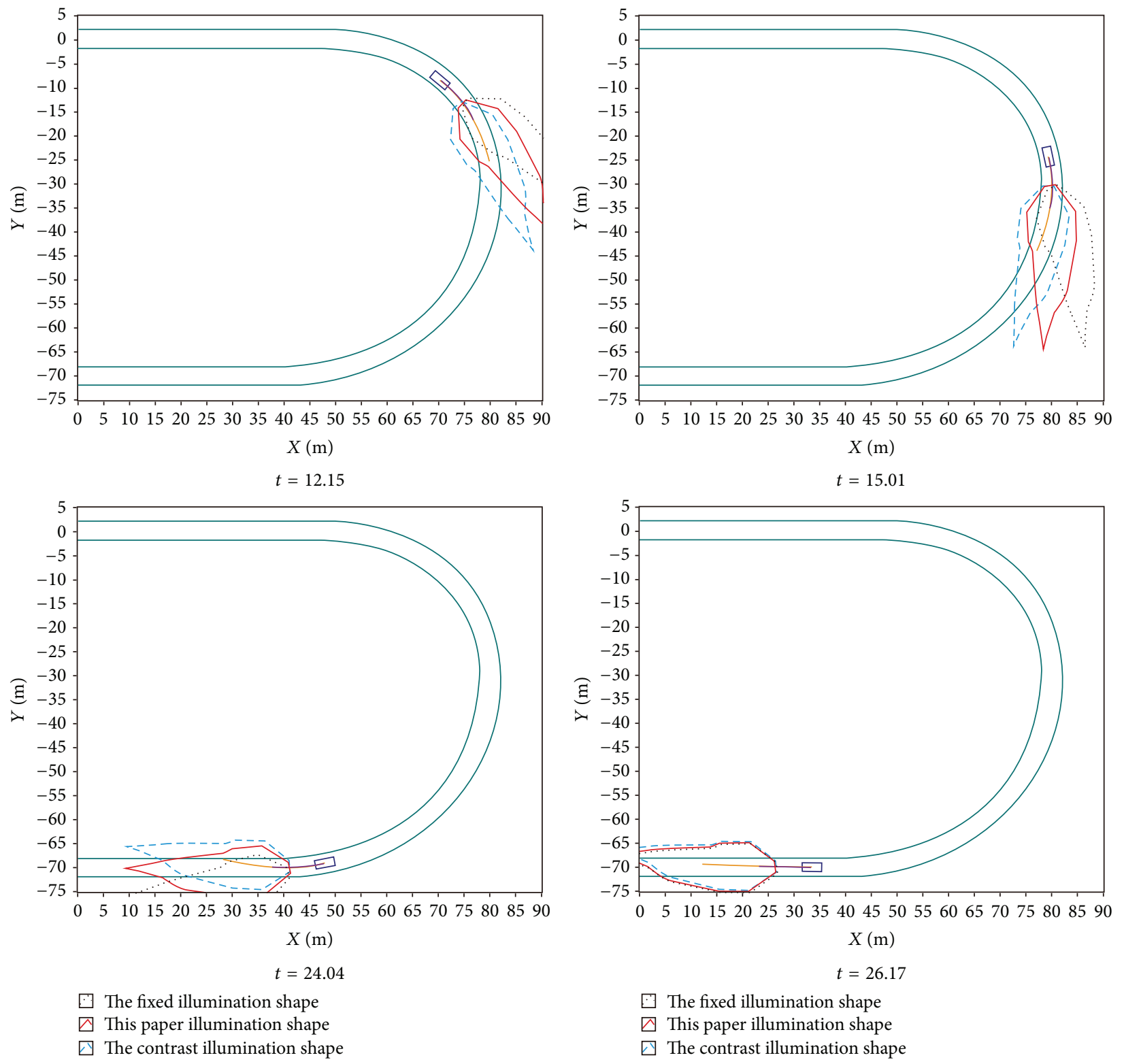

FIGURE 16: The front-lighting illumination area simulation schematic diagram 2.

deflection more precisely to ensure the front road's illumination effect well.

(2) Relative to the contrast algorithm, when the vehicle was in the bend and was going to be out of the bend, the headlamp's deflection angle of our algorithm was smaller; the headlamp's beam could cover the front road more well and make sure that the region the driver was interested in was in the central zone of the headlamp's beam which meant better illumination effect.

(3) When the vehicle was just in the bend and was out of the bend after a while, the headlamp's deflection angle of both algorithms was the same and their illumination effects were basically the same too.
In summary, the proposed algorithm could predict the vehicle's future track more precisely; on the basis of it, the headlamp's deflection angle was controlled to improve the front road's illumination condition effectively. Meanwhile, the proposed algorithm could make sure that the central zone of the headlamp's beam covered more regions which the driver was interested in.

\section{Conclusion}

The previous road experiment has found that the preview distance increased linearly with the velocity and the road curvature radius's increasing. Based on this rule, the precise preview distance was acquired by the eye tracker. The average preview time under the speed of $10-30 \mathrm{~km} / \mathrm{h}$ is $1.70 \mathrm{~s}$. Under 
the curvature radius of $(20-40) \mathrm{m}$, the average preview time is $1.44 \mathrm{~s}$. An empirical model of the preview distance was proposed which accorded with the driver's visual characteristics.

Based on the dynamic predict algorithm of the vehicle's future track using the driver's best preview acceleration model, the algorithm's fixed preview time was modified by road experiments and a full AFS algorithm was developed based on the algorithm. The future track's prediction of the proposed algorithm was precise.

Finally, by the cosimulation of CarSim and LabVIEW, the headlamp's illumination area and the vehicle's future track of different time were analyzed in detail in the bend driving process. The illumination effect was analyzed in two aspects and was compared with a mature nonpredictive algorithm. The result showed that the control effect was obvious and the algorithm had a good application prospect.

\section{Conflict of Interests}

The authors declare that there is no conflict of interests regarding the publication of this paper.

\section{Acknowledgments}

This paper is supported by the National Natural Science Foundation of China (50975120), Specialized Research Fund for the Doctoral Program of Higher Education (20120061110028), Jilin Provincial Research Foundation for Technology Guide (20130413058GH), and Program for Chang Jiang Scholars and Innovative Research Team in University (no. IRT1017), China. Thanks are due to them.

\section{References}

[1] ECE Regulation No. 123, Uniform Provisions Concerning the Approval of Adaptive Front-lighting System (AFS) for Motor Vehicles, 2007.

[2] L. Strambersky, M. Cejnek, and J. Martoch, "Fast Track-Static and Dynamic Bending Analysis," SAE Paper 2004-01-0440, 2004.

[3] R. Huang, Automobile Adaptive Lighting System Development, Dalian University of Technology, 2008.

[4] L. Lifu and D. Qian, "Research on the mathematical model of bending mode of vehicle adaptive front-lighting system," Aotomobile Technology, no. 10, pp. 43-46, 2010.

[5] X. Yu, Study on Automobile Adaptive Lighting Control System Dalian, University of Technology, 2009.

[6] Z. Deng, "The develop of AFS-adaptive frontlighting-system," China Light \& Lighting, no. 6, pp. 8-11, 2006.

[7] S. McLaughlin, J. Hankey, C. A. Green, and M. Larsen, “Target detection distances and driver performance with swiveling HID headlamps," SAE Paper 2004-01-2258, 2004.

[8] H. Rong, J. Gong, and J. Cao, "Research on key technology of AFS system auto electric parts," no. 5, pp. 15-18, 2008.

[9] W. Pu, "The profile and trends of Adaptive Front Lighting System (AFS)," Lamps and Lighting, no. 2, pp. 22-24, 2009.

[10] F. A.-K. Ibrahim, "Predictive Adaptive Front Lighting Algorithm for Branching Road Geometry: United States," Tech. Rep., US 7568822 B2, 2009.
[11] K. Ishiguro and Y. Yamada, "Control technology for bending mode AFS," SAE Paper 2004-01-0441, 2004.

[12] K.-H. Kim, D.-H. Yum, D.-K. Byeon, D.-Y. Kim, and D.-I. Lee, "Improving driver's visual field using estimation of curvature," in International Conference on Control, Automation and Systems (ICCAS '10), pp. 728-731, KINTEX, October 2010.

[13] M. F. Land, "Predictable eye-head coordination during driving," Nature, vol. 359, no. 6393, pp. 318-320, 1992.

[14] M. F. Land and D. N. Lee, "Where we look when we steer," Nature, vol. 369, no. 6483, pp. 742-744, 1994.

[15] M. Land and J. Horwood, "Which parts of the road guide steering?" Nature, vol. 377, no. 6547, pp. 339-340, 1995.

[16] M. Young, C. Chang, and Y. Chuang, "A study of drivers' viewing field when driving on curvy roads," in Proceedings of the the 3rd International Conference of Traffic \& Transport Psychology, Nottingham, UK, 2004.

[17] H. Zhang and X. Zhang, "Robust gain-scheduling gnergy-topeak control of vehicle lateral dynamics stabilisation," Vehicle System Dynamics, vol. 52, no. 3, pp. 309-340, 2013.

[18] Z. Shuai and H. Zhang, "Lateral motion control for fourwheel-independent-drive electric vehicles using optimal torque allocation and dynamic message priority scheduling," Control Engineering Practice, vol. 24, pp. 55-56, 2014.

[19] Z. Shuai and H. Zhang, "Combined AFS and DYC control of four-wheel-independent-drive electric vehicles over CAN network with time-varying delays," IEEE Transactions on Vehicular Technology, vol. 63, no. 2, pp. 591-602, 2014.

[20] Z. Gao and F. Gao, "Intelligent vhicle head lamp system simulating preview action of driver," CN 201010175695, China, 2010.

[21] X. Guan, Z. Gao, and K. Guo, "A hypothesis of steady preview and dynamic correction for driver model," Chinese Automotive Engineering, vol. 25, no. 3, pp. 19-22, 2003.

[22] Z. Gao, X. Guan, Q. Li et al., "Driver optimal preview longitudinal acceleration model," Automotive Engineering, vol. 24, no. 5, pp. 434-437, 2002.

[23] K. Guo, Vehicle Handling Dynamics Theory, Jiangsu Science and Technology Publishing House, Nanjing, China, 2011.

[24] K. Noh, D. Jung, H. Choi et al., "Development of ergonomic driver model considering human factors," SAE Paper 2007-013584, 2007. 


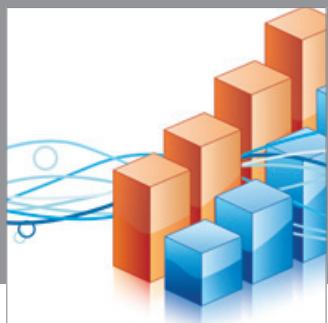

Advances in

Operations Research

mansans

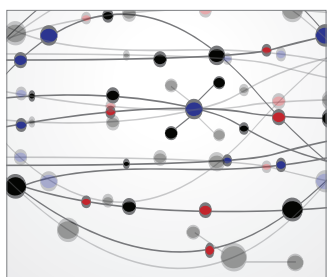

The Scientific World Journal
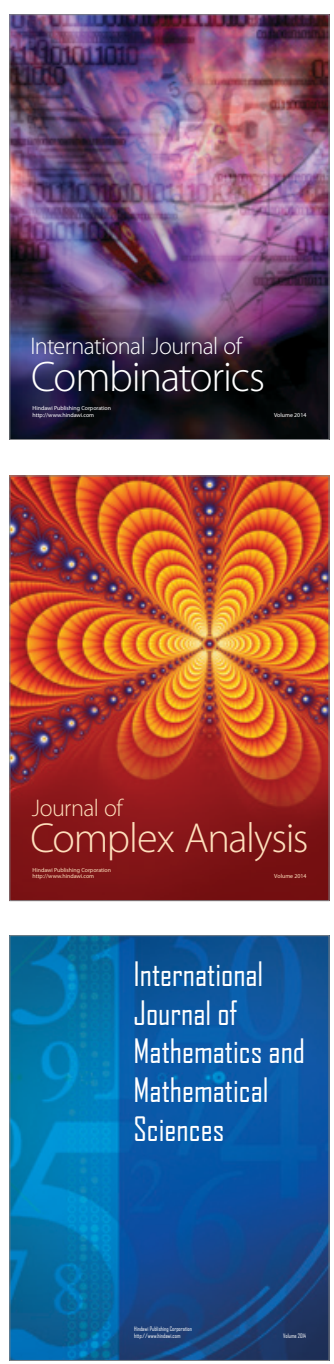
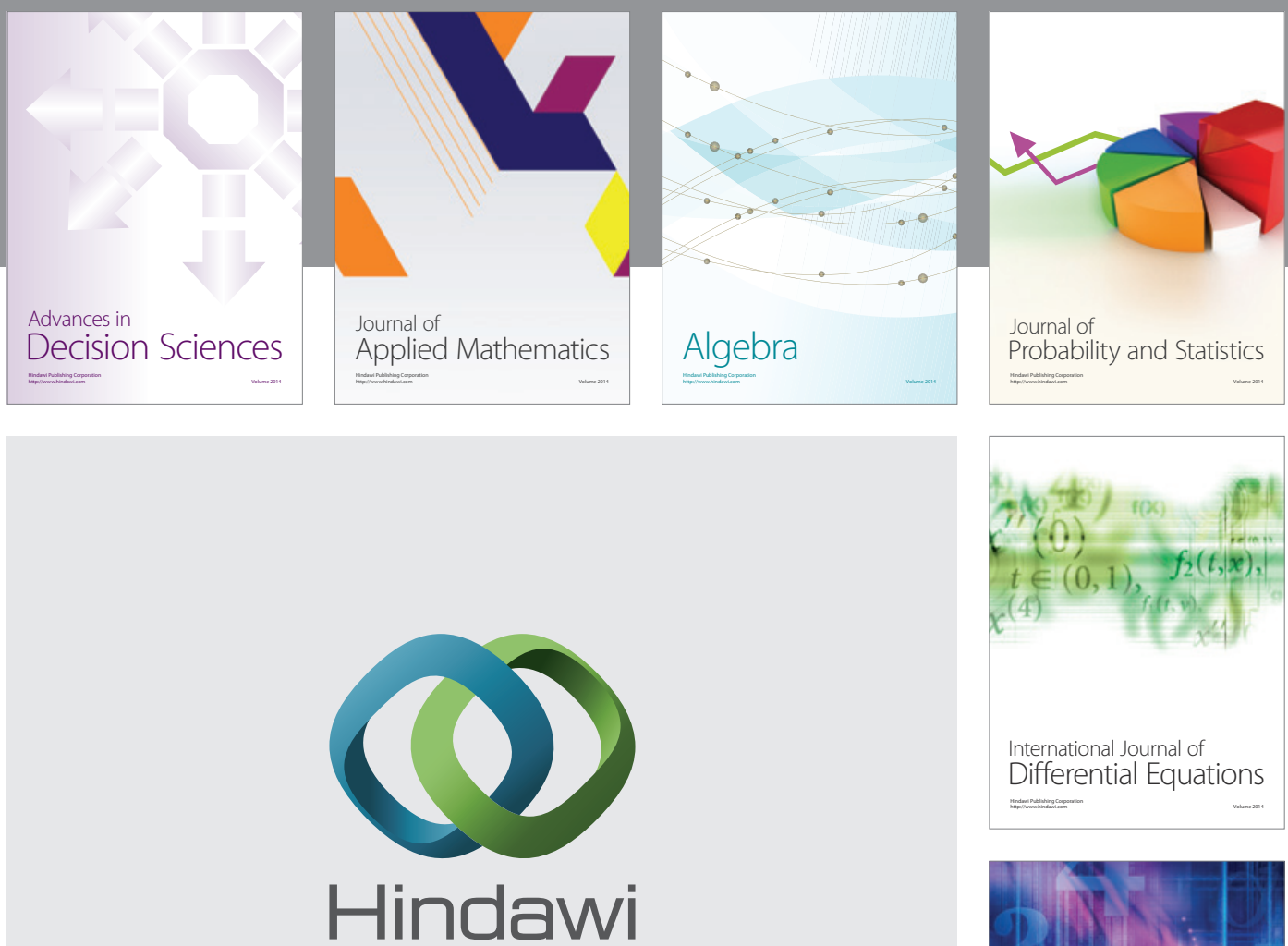

Submit your manuscripts at http://www.hindawi.com
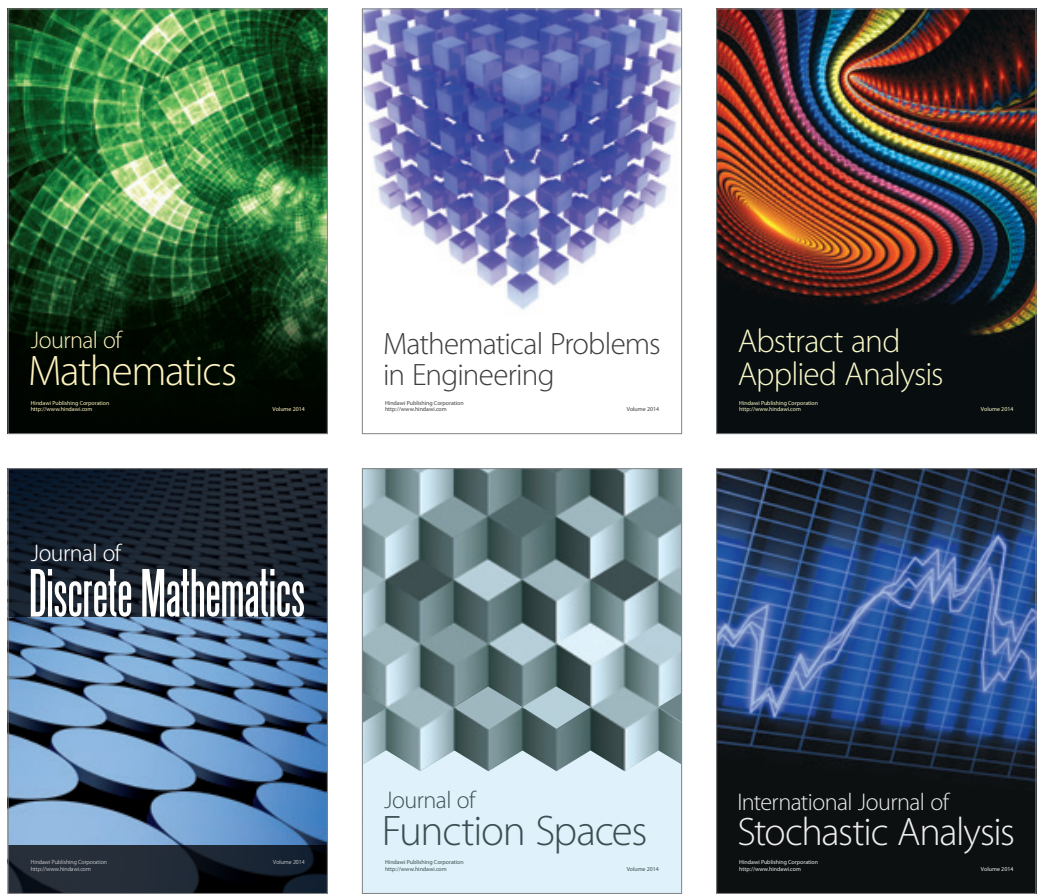

Journal of

Function Spaces

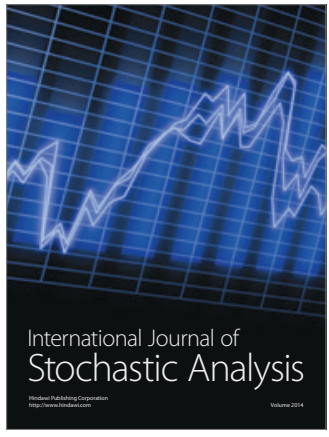

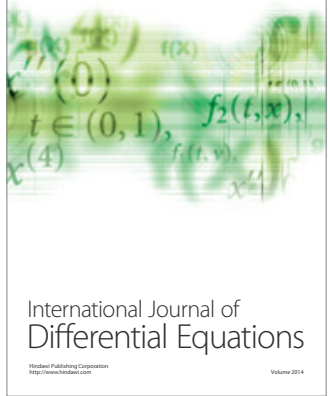
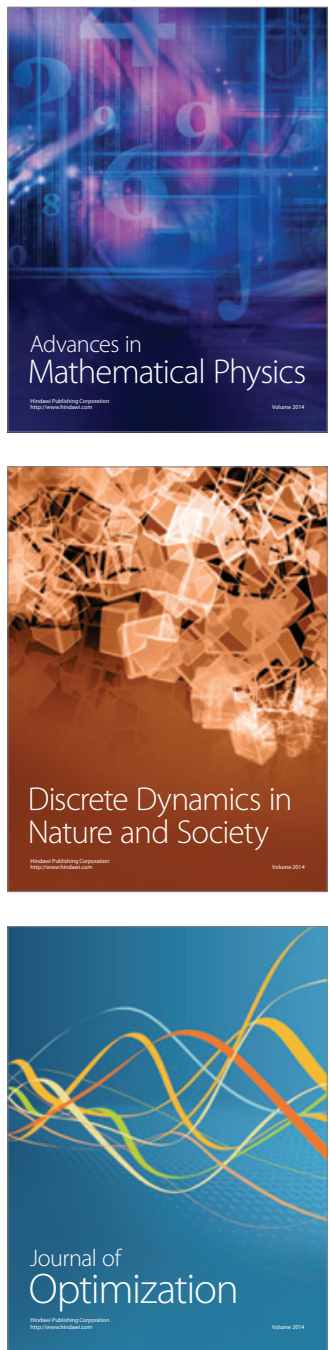